\title{
Celiac and non-celiac gluten sensitivity: a review on the association with schizophrenia and mood disorders
}

\author{
Brunetta Porcelli • Valeria Verdino • \\ Letizia Bossini · Lucia Terzuoli • Andrea Fagiolini
}

Received: 12 September 2014/ Accepted: 1 October 2014/Published online: 16 October 2014

(C) Springer International Publishing Switzerland 2014

\begin{abstract}
An association between many psychiatric and gluten-related disorders has been known for some time. In the case of schizophrenia and mood disorders, the major psychiatric disorders, there is much evidence, not without contradictions, of a possible association between schizophrenia and celiac disease. The association between mood disorders and gluten-related disorders, especially celiac disease, has only been studied for depression, often coupled with anxiety, and very recently for bipolar disorder. Since non-celiac gluten sensitivity is now known to be different from celiac disease, many studies have shown that gluten sensitivity is also associated with major psychiatric disorders. Here we review the literature on the association between schizophrenia/mood disorders and celiac disease/ gluten sensitivity, pointing out the differences between these associations.
\end{abstract}

Keywords Celiac disease - Gluten sensitivity ·

Psychiatric disorders

\section{Introduction}

An increased rate of autoimmune diseases has been demonstrated in individuals with psychiatric disorders such as

\section{B. Porcelli $(\bowtie) \cdot$ L. Terzuoli}

Biochemistry Division, Department of Medical Biotechnologies, Siena University, Polo Scientifico Universitario di San Miniato Via Alcide De Gasperi 2, 53100 Siena, Italy

e-mail: brunetta.porcelli@unisi.it

V. Verdino $\cdot$ L. Bossini $\cdot$ A. Fagiolini

Psychiatry Division, Department of Molecular and

Developmental Medicine, Siena University, Policlinico Le

Scotte Viale Bracci 1, 53100 Siena, Italy schizophrenia (SCZ) [1, 2] and mood disorders (MD) [3, 4]. Individuals diagnosed as having an autoimmune disease are also likely to be at increased risk of psychiatric disorders. Thus, the involvement of autoimmune mechanisms is increasingly held responsible for the pathogenesis of psychiatric disorders. Among autoimmune diseases, celiac disease (CD) has been described for years in association with SCZ [5-7], as celiac patients are felt to be at increased risk of schizophrenia. However, it has recently been discovered that the immune response to gluten in schizophrenic patients diverges from that in $\mathrm{CD}[8,9]$. As far as mood disorders are concerned, since they have not been systematically studied as disorders, their association with $\mathrm{CD}$ is neither conclusive nor systematic and is limited to depression, often coupled with anxiety. Moreover, when a positive statistical association between depression and celiac disease has been found, the percentage frequencies varied from 6 to $57 \%$ [10]. There is still a lack of consensus about the nature of this association, with two opposing schools of thought: the first posits that specific mechanisms are implied in a common pathogenesis, while the second considers depression not specifically linked to $\mathrm{CD}$ but rather a chance event [11]. Regarding bipolar disorder (BD), to date only a few studies have investigated its association with $\mathrm{CD}$. The first report showed increased risk of developing depression but not bipolar disorder in patients diagnosed with $\mathrm{CD}$, while prior depression and BD were positively associated with subsequent onset of $\mathrm{CD}$ [12]. In a recent study, celiac disease was not demonstrated to determine a statistically significant risk of bipolar disorder [4]. However, like for schizophrenia, recent research showed that immunological reactivity to gluten, different from $\mathrm{CD}$, can also be present in bipolar disorder [13-15]. This reactivity towards gluten is known as non-celiac gluten sensitivity, or more simply gluten sensitivity (GS), recently found to be different from CD [16]. 
Here we review the major literature on the association between schizophrenia/mood disorders and $\mathrm{CD} /$ gluten sensitivity, highlighting the differences between these associations.

Gluten-related disorders: new classification

A new nomenclature and classification for gluten-related disorders was recently proposed [16] to sort out the confusion created by new data on different reactions to gluten. Based on pathogenesis, gluten-related disorders can be divided into three types: (1) celiac disease, gluten ataxia and dermatitis herpetiformis, all with autoimmune pathogenesis; (2) wheat allergy, characterized by allergic mechanisms; (3) gluten sensitivity in which neither allergic nor autoimmune mechanisms are involved.

Though GS and CD cannot be distinguished clinically, the two conditions usually show several differences: even in the absence of epidemiological studies, GS is estimated to have prevalence six times higher than that of CD. Gluten sensitivity is also characterized by a lack of intestinal damage, negative CD serology (anti-tissue transglutaminase antibodies and anti-endomysial antibodies) and a possible presence of biomarkers of native gluten immune reaction (anti-gliadin antibodies) [17]. Moreover, while CD is strongly associated with specific human leukocyte antigen (HLA) class II genes, known as HLA-DQ2 and HLADQ8, these genes are present in only about $50 \%$ of patients with GS [18]. A recent study also demonstrated that gut permeability does not occur in GS, unlike in CD [19]. Thus, GS only seems to be characterized by activation of innate immune response, rather than adaptive response, which is responsible for detectable mucosal damage in CD. Finally, analysis of intestinal mucosal specimens from CD patients has demonstrated increased expression of IL-17A, lacking in GS patients [20].

On top of that, it has been observed that the prime manifestations of GS are often extra-intestinal, such as behavioral changes, bone or joint pain, muscle cramps, leg numbness, weight loss and chronic fatigue. This is why gluten sensitivity may easily go unrecognized and untreated, representing a risk factor for neurologic and psychiatric complications even greater than CD [21].

\section{Schizophrenia}

A connection between schizophrenia and gluten began to be noticed in the 1950s, as indicated by clinical cases like that reported by Bender [22]. During World War II, Dohan [23] sustained the hypothesis of this link by recording fewer first hospital admissions for SCZ in five countries. He correlated this phenomenon with decreased consumption of wheat and rye in wartime. The reduced supply of cereals seemed to determine a lower incidence of schizophrenia, suggesting that they were related.

Another observation was that a great number of admissions for SCZ occurred in countries like Ireland with higher availability of wheat and rye than in England and Wales [24].

Studies on the effect of elimination of gluten from the diet of SCZ patients further strengthened the existence of an association between gluten and schizophrenia. In fact, SCZ patients whose symptoms improved after introduction of a cereal- and milk-free diet showed an interruption or reversal of clinical improvement during wheat challenge [25].

At that time, the evidence pointed to a link between schizophrenia and gluten, and the authors suggested that celiac disease and SCZ had the same pathogenesis, namely the harmful effect of wheat. They hypothesized that the common clinical manifestations might signal a genetic link. This called for further studies to establish whether gluten consumption was a triggered genetic susceptibility, as suggested by Dohan [26]. Subsequent observations often provided contrasting results. Epidemiological studies, such as that of the National Danish Register [5], found that celiac disease occurred before onset of schizophrenia and that antibody-based diagnosis was a risk factor for SCZ. A higher than expected prevalence of certain autoimmune disorders (including CD) was also found [1] before onset of schizophrenia in patients diagnosed with this mental illness and their relatives. Almost at the same time, Ludvigsson [27] demonstrated that individuals with CD may be at increased risk of non-affective psychosis but not SCZ. However, analysis of data collected by the UK General Practice Research Database indicated that the risk of SCZ in patients diagnosed with autoimmune gastrointestinal disease (CD, ulcerative colitis and Crohn's disease) was no higher than in the general population [28]. Studies on the effect of eliminating gluten from the diet of schizophrenic patients, reviewed by Kalaydjian et al. [6], showed that psychotic symptoms only declined in a subset of patients. These results are therefore contradictory: on one hand they suggest a common autoimmune pathway for celiac disease and schizophrenia, but on the other they refute it.

Until a few years ago, CD and gluten sensitivity were considered synonymous for celiac disease. Uncertainty in disease classification and the absence of reliable methods, especially in older studies, may therefore explain these conflicting results. Some studies may also have been biased by the fact that that certain SCZ patients were not tested for the antibodies. However, this bias was overcome by studies in which antibody levels characterizing celiac disease were determined in schizophrenic patients. A study conducted in Israel to detect anti-endomysial antibodies (EMA) showed 
no difference between a group with chronic SCZ and a group of healthy controls [29]. Since EMA are very specific for celiac disease, this result could be interpreted as pointing to certain negativity for CD. However, since antitissue transglutaminase antibodies (a-tTG) and anti-gliadin antibodies (AGA) were not investigated, this result cannot exclude gluten sensitivity in this group of patients, as suggested by increasing evidence that GS is frequent in schizophrenia [8, 30].

Given the lack of consensus on the prevalence of true CD in schizophrenia, Samaroo et al. [9] tried to characterize the immune response to gluten in a subset of schizophrenic patients with elevated AGA titres. In this group, the elevation of AGA was not associated with increased prevalence of a-tTG, deamidated gliadin peptide (DGP) antibodies (another serological diagnostic marker of CD), or with celiac-related HLA genes. The authors concluded that these patients did not have celiac disease, but that SCZ patients probably have different mechanisms evoking an immune response against gluten, independent of transglutaminase and HLADQ2 and/or DQ8 molecules.

A longitudinal prospective study on antibody reactivity to gliadin, deamidated gliadin and tissue transglutaminase led to the same conclusions [8]. Patients with recent-onset psychosis showed higher levels of IgG and IgA AGA than individuals with multi-episode schizophrenia and individuals without a psychiatric history. These results confirmed those of Samaroo et al. [9]: the immunological pattern of schizophrenia diverged from that of celiac disease, since increased IgA a-tTG and IgG DGP antibodies were absent except for a marginal elevation in IgA a-tTG titre measured in the recent-onset psychosis group. Again, disease-related differences in the prevalence of DQ2/DQ8 alleles associated with $\mathrm{CD}$ were not found.

Cascella et al. [30] designed a study to evaluate the immunological pattern of a group of schizophrenic patients taking part in the Clinical Antipsychotic Trials of Intervention Effectiveness. From the serologic characterization of this patient sample, two groups emerged: one with elevated IgA AGA indicative of gluten sensitivity and the other with EMA and/or a-tTG plus IgA AGA, markers indicative of celiac disease. Thus the great majority of schizophrenic patients showed a dramatically higher prevalence of AGA antibodies than the comparison group, while reactivity to a-tTG was only mildly increased and reactivity to EMA was even lower. The discrepancy between the levels of a-tTG and EMA was recognized to be crucial, since they target the same antigen, and this indicated the need for further research. The authors suggested that the results may have been biased by the different assay techniques used: indirect immunofluorescence for EMA and ELISA for a-tTG. Another theory was that the low level of EMA determined the milder intestinal damage observed in GS.
To explain the discrepancy between the levels of a-tTG and EMA, Cascella et al. [30] screened the same population of schizophrenic patients for transglutaminase 6 antibodies (a-tTG-6) [31]. As gluten causes an immune response to $\mathrm{tTG}$, it was reasonable to suppose that $\mathrm{tTG}$ isoforms other than tTG2 could be involved, for instance tTG-6, which would not cause intestinal but psychiatric manifestations. The authors looked for a-tTG-6 antibodies, which are primarily expressed in the brain and are reported to be elevated in gluten ataxia; they observed increased prevalence in SCZ patients. A reasonable explanation is that gluten may reach the brain through a breach in the blood-brain barrier and stimulate an immune response similar to that observed towards intestinal mucosa, leading neurons to expose tTG-6. As well, gluten seems to trigger release of zonulin, a modulator of the blood-brain barrier and the precursor for haptoglobin-2, an inflammatory marker found elevated in a subgroup of schizophrenic patients. This could explain increased blood-brain barrier permeability, allowing passage of gluten peptides and activated inflammatory cells. It also suggests the potential implications of a gluten-free diet (GFD) for the treatment of schizophrenia.

A recent study [32] analyzed a group of schizophrenic patients before they started a GFD, distinguishing CD from GS patients on the basis of antibodies (positivity for a-tTG or EMA and for AGA, respectively). Their response to the diet was tested by evaluating psychotic symptoms. The diet seemed to improve symptoms and extrapyramidal effects, without interfering with the therapy or the behavior of these patients. To date, this is the first study concerning elimination of gluten from the diet of patients previously screened for celiac disease and gluten sensitivity by serological markers. The results are encouraging, though the sample and duration of the trial were not sufficient for definitive proof, especially in case of GS, where the data were insufficient to discern how long it takes to see an improvement.

\section{Mood disorders}

There is still lack of conclusive and systematic results about association between mood disorders and celiac disease. Many authors found a positive statistical association with celiac disease [12, 33-36] while others did not [3740]. However, these results were limited to depressive symptoms, which were frequently investigated in association with anxiety, and bipolar disorder has seldom been studied [13]. Regarding a possible association between mood disorders and gluten sensitivity, there have been no systematic studies. The few studies are limited to the association bipolar disorders/gluten sensitivity [13-15]. 
Unipolar depression

In 1932, Thaysen observed peculiar fatigue in patients with intestinal complaints and in 1950, Dicke posited that gluten-free diet improved the mood of adult celiacs. In 1956, Dayne described improved behavior in celiac children on GFD and noticed that adults not compliant with the dietdeveloped problems like headache, insomnia and depression [41]. In 1970, Goldberg studied a group of 80 patients with diseases of the small intestine by standardized psychiatric assessment, finding that $34 \%$ had minor affective disorders [42]. The main risk factors he identified for comorbidity of idiopathic steatorrhoea and psychiatric illness were a positive family or personal psychiatric history and depressive personality traits prior to onset of small intestinal pathology. The author suggested a genetic link between psychiatric illness and the enzyme defect suspected to be responsible for steatorrhoea.

Later evidence supported the central role of malabsorption, believed to cause reduced central metabolism in the monoamine pathways [43, 44]. Hernanz and Polanco [45] sustained this theory, observing impaired levels of tryptophan in blood samples of untreated children with celiac disease. Nevertheless, malabsorption seemed unable by itself to determine sufficient depletion of tryptophan for psychiatric disorders, such as lifetime depressive and disruptive behaviour, observed in association with $\mathrm{CD}$ in a group of adolescents [46]. Thus, pro-inflammatory cytokines, such as IFN- $\gamma$, produced by activated T-cells in response to ingestion of gluten, were considered to contribute massively to serotonin dysfunction, affecting the kynurenine-niacin pathway and enhancing the hypothalamus-pituitary-adrenal axis.

Carta et al. [33] were the first to evaluate a possible role of autoimmunity in the pathogenesis of mood disorders, suggested by the finding of anti-thyroid peroxidase (antiTPO) antibodies in celiac patients where major depressive disorder, dysthymic disorder and panic disorder had significantly high frequency. Subclinical thyroid disease was suspected to be a risk factor for these psychiatric disorders. The authors suggested that cytokines produced in immune reactions may exert an effect on the hypothalamic-pituitary-adrenal axis, also affecting other brain circuits involved in mood control. They supposed that common neuroendocrine dysregulation partook in the pathogenesis of affective disorders and autoimmune diseases. In another study they showed that recurrent brief depression, diagnosed according to ICD-10 criteria, had even higher prevalence than major depression in celiac patients [47].

In celiac patients undergoing single-photon emission computed tomography, Addolorato et al. [48] observed that regional cerebral blood flow was more abnormal in untreated celiacs than in healthy controls, while there was no difference in cerebral perfusion between treated celiac patients and healthy controls.

Ciacci et al. [35] evaluated a sample of 100 celiac patients, 92 normal controls and 48 with chronic persistent hepatitis by means of a modified version of the Zung SelfRating Depression Scale and concluded that depression was a feature of $\mathrm{CD}$ unaffected by age at diagnosis, duration of illness or compliance/non-compliance with diet, whereas personal psychological resources appeared to be fundamental in developing or maintaining depression. In a general population-based cohort study in Sweden, Ludviggson et al. [12] also found increased risk of subsequent depression in individuals suffering from celiac disease. Siniscalchi et al. [36] observed that fatigue was common in celiac patients and was not related to GFD, though it seemed less intense in treated patients. They found that celiac patients on GFD showed stronger depression symptoms than celiacs on a normal diet. Data gathered by Hauser [49] showed that the duration of the GFD was not related to depressed mood, and this was the same for anxiety. Nonetheless, while CD patients on GFD were not more depressed than controls, female CD patients on GFD seemed at increased risk of developing anxiety disorder.

If on one hand there are encouraging results, like those just cited, supporting the hypothesis that depression and anxiety are related to celiac disease, on the other hand different studies found no significant association. For instance Cicarelli et al. [37] found that, although depression appeared to be associated with $\mathrm{CD}$, as found in previous studies, the mood symptoms of celiac patients were the result of dysthymic disorder, not depression, diagnosed by DSM IV.

According to Fera et al. [38], affective disorders in celiacs may be ascribed to difficulties in adjusting to the chronic nature of the disease rather than directly to the disease itself. Accomando et al. [39] administered two depression assessment questionnaires to $\mathrm{CD}$ patients on GFD for more than 2 years to determine the existence of a relationship between GFD and depression. The results failed to show any difference between celiac patients and healthy controls. Roos et al. [40] used the Psychological General Well-being index to assess celiac patients on GFD for many years. Long-treated adult patients showed no difference in psychological well-being with respect to controls, suggesting that signs of depressed mood are not a feature of well-treated celiac disease.

The state of current understanding of this topic is extremely confusing. A meta-analysis [11] comprising results from reports on anxiety and depression in adult celiac disease failed to show a higher frequency of depression in celiac patients than in patients with other chronic illnesses. 
Bipolar disorder

In 2011, the association of bipolar disorder with celiac disease and gluten sensitivity was investigated for the first time by Dickerson et al. [13]. Antibody assessment of BD patients showed significantly higher levels of IgG AGA than in controls. There was also an eloquent increase in IgG DGP antibodies in the BD group, whereas IgA AGA and $\mathrm{IgA} / \operatorname{IgG}$ a-tTG levels did not differ between the two groups. Dickerson et al. [14] continued to study the longitudinal trend of these antibodies in patients admitted during a manic episode and at 6-month follow-up. Only IgG AGA was significantly elevated in the acutely manic group. However, these levels were not significantly different from those of controls at 6-month follow-up. Among manic individuals, elevated levels at follow-up were significantly associated with re-hospitalization in the 6-month follow-up period, suggesting a correlation between GS and the clinical course of manic symptoms.

Another clinical study screened a sample of 103 psychiatric inpatients [15] for a wide spectrum of autoantibodies, finding significantly higher IgG AGA levels in inpatients than controls. Bipolar disorder appeared to be the disorder most associated with autoantibodies.

\section{Comparison of schizophrenia/mood disorders and CD/ GS associations}

Generally, the association of gluten sensitivity with schizophrenia has been more systematically investigated than its association with mood disorders. In bipolar disorder, a-tTG has never been found significantly different from controls [13], while in schizophrenia IgA a-tTG [30] and IgG a-tTG [8] have been found slightly higher than in controls. However, the increase in IgA a-tTG [30] was not accompanied by an increase in levels of anti-endomysial antibodies (EMA), excluding the possibility of celiac disease. Regarding schizophrenia, antibodies to gliadin did not show significant affinity for deamidated gliadin peptides, again excluding the possibility of celiac disease but suggesting a response to gluten with different antigen specificity in a majority of gluten-sensitive individuals with schizophrenia [9]. On the contrary, Dickerson et al. [13] found evidence of increased levels of IgG DGP in BD patients, though this result was not replicated in the later study [14]. There have also been few studies on bipolar disorder and gluten sensitivity, however, they all yielded results consistent with an increase in IgG AGA levels [1315], whereas some studies on schizophrenia and gluten sensitivity only found an increase in IgA AGA [30, 50].

The effect of elimination of gluten from the diet has never been studied in bipolar disorder, whereas for schizophrenic patients it is described throughout the literature [6]. A recent study [32] tested response to a glutenfree diet in a group of SCZ patients who were previously considered to have celiac disease or gluten sensitivity on the basis of antibody assessment.

\section{Discussion}

The recent characterisation of non-celiac gluten sensitivity shows that like celiac disease, this gluten-related disorder is also associated with major psychiatric disorders. In this review of the literature on the association between SCZ/ MD and CD/GS, we found that for many years, studies on the association between schizophrenia and celiac disease provided contrasting results [5-7, 28, 29]. Celiac patients were thought to be at higher risk of developing SCZ than the general population [5-7]. Studies on the association between mood disorders and celiac disease did not use a systematic approach and provided contrasting results [12, 33-40]. Furthermore, these results were always limited to depressive symptoms, frequently investigated in association with anxiety, while bipolar disorder was studied to a lesser extent [13].

With regard to the association between these major psychiatric disorders and gluten sensitivity, the results are not always concordant, essentially due to the fact that despite a body of data on GS, this disorder is not yet completely characterized and there are still no serological markers for its diagnosis.

Regarding the association between schizophrenia and gluten sensitivity, most recent studies agree on the involvement of gluten sensitivity rather than celiac disease in a subgroup of SCZ patients [8, 9, 30, 50, 51]. As far as the association between mood disorders and gluten sensitivity is concerned, no systematic studies have been conducted. Only bipolar disorder has been studied, assessing antibodies in a sample previously diagnosed with psychiatric disease [13-15]. To our knowledge there have been no studies in which GS was evaluated in patients with confirmed unipolar depression.

A deeper understanding of the immune alterations documented in bipolar disorder and schizophrenia is currently impossible. Many theories have been put forward. Focusing on bipolar disorder, immune activation is not likely to directly affect the brain [14]. Altered mechanisms of absorption of food protein and therefore processing of food antigen are possibly involved, in line with documented heightened immune activation towards milk caseins [52] in this disorder. It is also worth noting the increasing evidence in support of the role played by infections, more than autoimmune diseases, in the pathogenesis of mood disorders [4]. Infection by Toxoplasma 
gondii [53] and other neurotropic pathogens, such as influenza and coronavirus, is associated with MD [54]. In schizophrenia, Toxoplasma is suspected to directly affect the brain and the action of cytokines. Its passage may occur by alterations in intestinal permeability due to mimicry of enterocyte epitopes by gliadin [15]. A recent study has demonstrated that Toxoplasma gondii [55] infections raise gluten antibody levels in mice, suggesting that it may alter transcellular passage of gluten peptides. Alterations in commensal gut microbiota have also been suggested to dysregulate the immune system, leading to production of antibodies [15]. Another recent study found that levels of SCD14, a marker of translocation of commensal microbiota, were significantly correlated with anti-tissue transglutaminase IgG in bipolar disorder [56]. We currently do not know whether alterations mainly involve intestinal breakdown, absorption, immune response to gliadin, autoantibody cross-reactivity, production of neuroactive peptides in altered digestion processes [14] or activation of $\mathrm{T}$ cell bystander and consequently B cells with antibody production [13, 52]. Altered permeability, gut microbiota alterations and infections could therefore all be putative pathogenetic mechanisms involved in SCZ and MD.

\section{Conclusions}

Further studies on the association between schizophrenia/ mood disorders and immune reaction to gluten are warranted to establish the threshold for therapeutic intervention, as only one study has systematically studied the effect of a gluten-free diet in schizophrenic GS and CD patients [32]. New insights into gluten sensitivity have helped to understand recent results showing immune responses to gluten proteins in certain psychiatric disorders but the different findings are still incoherent. Studying these associations provides a model for understanding the pathogenetic mechanisms behind psychiatric disorders, the neurobiology of which is still far from understood.

\section{Conflict of interest None.}

Human and animal rights This article does not contain any studies with human participants or animals performed by any of the authors.

Informed consent For this type of study formal consent is not required.

\section{References}

1. Eaton WW, Byrne M, Ewald H, Mors O, Chen C-Y, Agerbo E, Montersen PB (2006) Association of schizophrenia and autoimmune diseases: linkage of Danish national registers. Am J Psychiatry 163:521-528

2. Benros ME, Nielsen PR, Nordentoft M, Eaton WW, Dalton SO, Mortensen PB (2011) Autoimmune diseases and severe infections as risk factors for schizophrenia: a 30-year population-based register study. Am J Psychiatry 168:1303-1310

3. Eaton WW, Pedersen MG, Nielsen PR, Mortensen PB (2010) Autoimmune diseases, bipolar disorder, and non-affective psychosis. Bipolar Disord 12:638-646

4. Benros ME, Waltoft BL, Nordentoft M, Ostergaard SD, Eaton WW, Krogh J et al (2013) Autoimmune diseases and severe infections as risk factors for mood disorders: a nationwide study. JAMA Psychiatry 70:812-820

5. Eaton WW, Mortensen PB, Agerbo E, Byrne M, Mors O, Ewald H (2004) Coeliac disease and schizophrenia: population based case control study with linkage of Danish national registers. BMJ 328:438-439

6. Kalaydjian AE, Eaton W, Cascella N, Fasano A (2006) The gluten connection: the association between schizophrenia and celiac disease. Acta Psychiatr Scand 113:82-90

7. Chen SI, Chao YL, Chen CY, Chang CM, Wu EC, Wu CS et al (2012) Prevalence of autoimmune diseases in in-patients with schizophrenia: nationwide population-based study. Br J Psychiatry 200:374-380

8. Dickerson F, Stallings C, Origoni A, Vaughan C, Khushalani S, Leister F, Yang S, Krivogorsky B, Alaedini A, Yolken R (2010) Markers of gluten sensitivity and celiac disease in recent-onset psychosis and multi-episode Schizophrenia. Biol Psychiatry 68:100-104

9. Samaroo D, Dickerson F, Kasarda D, Green P, Briani C, Yolken RH, Alaedini A (2010) Novel immune response to gluten in individuals with Schizophrenia. Schizophr Res 118:248-255

10. Garud S, Leffler D, Dennis M et al (2009) Interaction between psychiatric and autoimmune disorders in coeliac disease patients in the northeastern United States. Aliment Pharmacol Ther 29:898-905

11. Smith DF, Gerdes LU (2012) Meta-analysis on anxiety and depression in adult celiac disease. Acta Psychiatr Scand 125:189-193

12. Ludvigsson JF, Reutfors J, Osby U, Ekbom A, Montgomery SM (2007) Coeliac disease and risk of mood disorder-A general population based cohort study. J Affect Disord 99:117-126

13. Dickerson F, Stallings C, Origoni A, Vaughan C, Khushalani S, Alaedini A, Yolken R (2011) Markers of gluten sensitivity and celiac disease in bipolar disorder. Bipolar Disord 13:52-58

14. Dickerson F, Stallings C, Origoni A, Vaughan C, Khushalani S, Yolken R (2012) Markers of gluten sensitivity in acute mania: a longitudinal study. Psychiatry Res 196:68-71

15. Sidhom O, Laadhar L, Zitouni M, Ben Alaya N, Rafrafi R, KallelSellami M et al (2012) Spectrum of autoantibodies in Tunisian psychiatric inpatients. Immunol Invest 41:538-549

16. Sapone JC Bai, Ciacci C, Dolinsek J, Green PHR, Hadjivassiliou M, Kaukinen K et al (2012) Spectrum of gluten-related disorders: consensus on new nomenclature and classification. BMC Med 10:13-24

17. Hadjivassiliou M, Williamson CA, Woodroofe N (2004) The immunology of gluten sensitivity: beyond the gut. Trends Immunol 25:578-582

18. Bizzaro N, Tozzoli R, Villalta D, Fabris M, Tonutti E (2010) Cutting-edge issues in celiac disease and in gluten intolerance. Clin Rev Allergy Immunol 42:279-287

19. Sapone A, Lammers KM, Casolaro V, Cammarota M, Giuliano MT et al (2011) Divergence of gut permeability and mucosal immune gene expression in two gluten-associated conditions: celiac disease and gluten sensitivity. BMC Med 9:23

20. Sapone A, Lammers KM, Mazzarella G, Mikhailenko I, Cartenì M, Casolaro V, Fasano A (2010) Differential mucosal IL-17 
expression in two gliadin-induced disorders: gluten sensitivity and the autoimmune enteropathy celiac disease. Int Arch Allergy Immunol 152:75-80

21. Jackson JR, Eaton WW, Cascella NG, Fasano A, Kelly DL (2012) Neurologic and psychiatric manifestations of celiac disease and gluten sensitivity. Psychiatr Q 83:91-102

22. Bender L (1953) Childhood schizophrenia. Psychiat Q 27:663-681

23. Dohan FC (1966) Wheat "consumption" and hospital admissions for schizophrenia during World War II. A preliminary report. Am J Clin Nutr 18:7-10

24. Walsh D (1973) Coeliac disease and Schizophrenia. Br Med J $2: 242$

25. Singh MM, Kay S (1976) Wheat gluten as a pathogenic factor in schizophrenia. Science 191:401-402

26. Dohan FC (1973) Coeliac disease and Schizophrenia. Br Med J 3:51-52

27. Ludvigsson JF, Osby U, Ekbom A, Montgomery SM (2007) Coeliac disease and risk of schizophrenia and other psychosis: a general population cohort study. Scand J Gastroenterol 42:179-185

28. West J, Logan RF, Hubbard RB, Card TR (2006) Risk of schizophrenia in people with coeliac disease, ulcerative colitis and Crohn's disease: a general population-based study. Aliment Pharmacol Ther 23:71-74

29. Peleg R, Ben-Zion ZI, Peleg A, Gheber L, Kotler M et al (2004) "Bred madness" revisited: screening for specific celiac antibodies among schizophrenia patients. Eur Psychiatry 19:311-314

30. Cascella N, Kryszak D, Bhatti B, Gregory P, Kelly DL, Mc Evoy JP, Fasano A, Eaton W (2011) Prevalence of celiac disease and gluten sensitivity in the United States clinical antipsychotic trials of intervention effectiveness study population. Schizophr Bull 37:94-100

31. Cascella NG, Santora D, Gregory P, Kelly DL, Fasano A, Eaton WW (2013) Increased prevalence of transglutaminase 6 antibodies in sera from schizophrenia patients. Schizophr Bull 39:867-871

32. Jackson J, Eaton W, Cascella N, Fasano A, Warfel D, Feldman S, Richardson C, Vyas G, Linthicum J, Santora D, Warren KR (2012) A gluten-free diet in people with schizophrenia and antitissue transglutaminase or anti-gliadin antibodies. Schizophr Res $140: 262-263$

33. Carta MG, Hardoy MC, Boi MF et al (2002) Association between panic disorder, major depressive disorder and celiac disease: a possible role of thyroid autoimmunity. J Psychosom Res 53:789-793

34. Addolorato G, Mirijello A, DAngelo C et al (2008) State and trait anxiety and depression in patients affected by gastrointestinal diseases: psychometric evaluation of 1641 patients referred to an internal medicine outpatient setting. Int $\mathrm{J}$ Clin Pract 62:1063-1069

35. Ciacci C, Iavarone A, Mazzacca G, De Rosa A (1998) Depressive symptoms in adult celiac disease. Scand J Gastroenterol 33:247-250

36. Siniscalchi M, Iovino P, Tortora R et al (2005) Fatigue in adult coeliac disease. Aliment Pharmacol Ther 22:489-494

37. Cicarelli G, Della RG, Amboni M et al (2003) Clinical and neurological abnormalities in adult celiac disease. Neurol Sci 24:311-317

38. Fera T, Cascio B, Angelini G, Martini S, Sategna Guidetti C (2003) Affective disorders and quality of life in adult coeliac disease patients on a gluten-free diet. Eur J Gastroenterol Hepatol 15:1287-1292
39. Accomando S, Fragapane ML, Montaperto D, Trizzino A, Amato GM, Calderone F et al (2005) Coeliacdisease and depression: two related entities? Dig Liver Dis 37:298-299

40. Roos S, Karner A, Hallert C (2006) Psychological well-being of adult coeliac patients treated for 10 years. Dig Liver Dis 38:177-180

41. De Fazio P, Ruberto S, Barbuto E, Sirianni M, Caglioti F, Garcia CS (2011) Disturbi Psichiatrici e Malattia Celiaca. In: Corposanto C (ed) Celiachia, malattia sociale. Un approccio multidisciplinare alle intolleranze alimentari. Milano, Franco Angeli, pp 85-105

42. Goldberg D (1970) A psychiatric study of patients with diseases of the small intestine. Gut 11:459-465

43. Hallert C, Astrom J (1982) Psychic disturbances in adult coeliac disease II. Psychological findings. Scand J Gastroenterol 17:21-24

44. Hallert C, Astrom J, Sedvall G (1982) Psychic disturbances in adult coeliac disease. III. Reduced central monoamine metabolism and signs of depression. Scand J Gastroenterol 17:25-28

45. Hernanz A, Polanco I (1991) Plasma precursor amino acids of central nervous system monoamines in children with coeliac disease. Gut 32:1478-1481

46. Pynnonen PA, Isometsa ET, Aronen ET et al (2004) Mental disorders in adolescents with celiac disease. Psychosomatics 45:325-335

47. Carta MG, Hardoy MC, Usai P, Carpiniello B, Angst J (2003) Recurrent brief depression in celiac disease. J Psychosom Res 55:573-574

48. Addolorato G, Di Giuda D, De Rossi G, Valenza V et al (2004) Regional cerebral hypoperfusion in patients with celiac disease. Am J Med 116:312-317

49. Hauser W, Janke KH, Klump B et al (2010) Anxiety and depression in adult patients with celiac disease on a gluten-free diet. World J Gastroenterol 16:2780-2787

50. Jin SZ, Wu N, Xu Q, Zhang X, Ju GZ, Law MH, We IJ (2012) A study of circulating gliadin antibodies in schizophrenia among a Chinese population. Schizophr Bull 38:514-518

51. Okusaga O, Yolken RH, Langenberg P, Sleemi A, Kelly DL, Vaswani D et al (2013) Elevated gliadin antibody levels in individuals with schizophrenia. World J Biol Psychiatry 14:509-515

52. Severance EG, Dupont D, Dickerson FB, Stallings CR, Origoni AE, Krivogorsky B et al (2010) Immune activation by casein dietary antigens in bipolar disorder. Bipolar Disord 12:834-842

53. Hamdani N, Daban-Huard C, Lajnef M, Richard JR, Delavest M, Godin O et al (2013) Relationship between Toxoplasma gondii infection and bipolar disorder in a French sample. J Affect Disord 148:444-448

54. Okusaga O, Yolken RH, Langenberg P, Lapidus M, Arling TA, Dickerson FB et al (2011) Association of seropositivity for influenza and coronaviruses with history of mood disorders and suicide attempts. J Affect Disord 130:220-225

55. Severance EG, Kannan G, Gressitt KL, Xiao J, Alaedini A, Pletnikov MV, Yolken RH (2012) Anti-gluten immune response following Toxoplasma gondii infection in mice. PLoS ONE 7:e50991. doi:10.1371/journal.pone.0050991

56. Severance EG, Gressitt KL, Stallings CR, Origoni AE, Khushalani S, Leweke FM, Dickerson FB, Yolken RH (2013) Discordant patterns of bacterial translocation markers and implications for innate immune imbalances in schizophrenia. Schizophr Res 148:130-137 